\title{
Encore un Oui à l'actualisation de la procréation médicalement assistée
}

\author{
Bruno Imthurna, Christian De Geyter ${ }^{b}$ \\ ${ }^{a}$ membre du Comité de la SSGO; ${ }^{\text {b }}$ Médecin-chef, Hôpital Universitaire de Bâle
}

En juin 2016, le peuple se prononcera encore une fois sur l'avenir de la procréation médicalement assistée. Après la décision du peuple et des cantons d'approuver l'année dernière à une grande majorité l'adaptation de l'article 119 de la Constitution fédérale, la Société suisse de gynécologie et d'obstétrique (SSGO gynécologie suisse) s'engage résolument en faveur de la révision de la Loi sur la procréation médicalement assistée. Cette révision permettra en effet d'optimiser la prise en charge de milliers de couples qui ont des difficultés à concrétiser leur désir d'enfant, de réduire le nombre des grossesses multiples et d'éviter des interruptions de grossesse.

\section{Historique}

En juin 2015, le peuple et les cantons ont adopté à une grande majorité (62\% des voix et 18,5 cantons favorables) la modification de l'article 119 de la Constitution fédérale. Ce plébiscite ouvre la voie à une révision [1] de la très restrictive Loi sur la procréation médicalement assistée (LPMA), en vigueur depuis 2001, et permet un retour à un niveau en partie déjà atteint depuis plusieurs décennies par la plupart des pays européens (tab. 1 [2]). Elaborée à la fin des années nonante et en vigueur depuis janvier 2001, la LPMA est en effet aujourd'hui dépassée par les progrès de la médecine et entraîne, contrairement à l'intention initiale, des étapes de traitement et des complications inutiles comme des grossesses multiples à risque. Grâce à la révision de la loi, il sera possible de les éviter.

Parallèlement à l'adaptation de l'article constitutionnel 119, le Conseil fédéral et le Parlement ont décidé et voté la révision de la LPMA fin 2014 [3, 4]. Avec cette procédure exceptionnelle, le peuple savait en toute transparence dès juin 2015 - contrairement aux votations constitutionnelles habituelles - l'impact de son vote sur la loi. C'est aussi la raison pour laquelle le débat préalable à la votation ne s'est pas concentré sur l'adaptation plutôt abstraite de la Constitution fédérale, mais sur les modifications concrètes de la LPMA. Malgré un oui largement majoritaire en juin 2015, le Parti évangélique suisse (PEV), qui avait déjà fait campagne contre la modification de l'article 119, a saisi le référendum contre la révision de la LPMA appelant ainsi le peuple suisse à se prononcer de nouveau le 5 juin prochain.

Quelles sont les principales conséquences de la révision de la Loi sur la procréation médicalement assistée, décidée par le Conseil fédéral et le Parlement?

Optimiser la prise en charge de milliers de couples qui chaque année ont des difficultés à concrétiser leur désir d'enfant

Selon la LPMA actuellement en vigueur, le nombre d'embryons imprégnés pouvant être développés hors du corps de la femme, entre la fusion d'ovules et de spermatozoïdes au jour $\mathrm{O}$ (insémination) et le constat au microscope de la fécondation le lendemain (jour 1), ne peut être supérieur à trois ("règle des trois»). La Suisse est le seul pays au monde à connaître une telle restriction. La législation en Allemagne est certes similaire mais contrairement à la nôtre, le respect de ce plafond n'est pas obligatoire et le non-respect de la «règle des trois» n'est pas punissable. Par ailleurs, de vifs débats remettent en question la pertinence de cette règle [5]. Avec la révision de la LPMA, la "règle des trois» ne sera pas totalement abandonnée mais remplacée par une «règle des douze» qui tient compte des inquiétudes exprimées en Suisse (art. 17, al. 1 LPMA).

Pour apprécier correctement cette «règle des douze», il convient de rappeler que $50 \%$ des embryons imprégnés dégénèrent naturellement entre le jour 1 (fécondation) 


\section{Nouvelle loi sur la procréation médicale- ment assistée: une prise en charge optimale, moins d'embryons surnuméraires*}

\section{Aujourd'hui}

Prélèvement de 10 ovules en moyenne addition de spermatozoïdes.

\section{Jour 0}

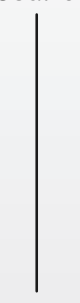

Jour 1

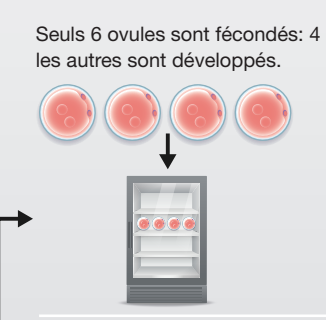

2 ovules développés sont implantés dans l'utérus.

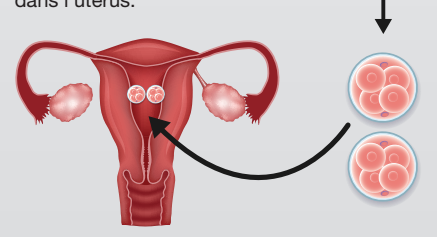
précision la viabilité des ovules transférés le premier jour, on implante en règle générale deux embryons, avec les risques de grossesse multiple que cette pratique comporte. En outre, le transfert d'embryons non viables rend souvent nécessaires plusieurs cycles de traitement.
Comme il est difficile de déterminer avec

\section{Après adoption de la loi}

Prélèvement de 10 ovules en moyenne et addition de spermatozoïdes.

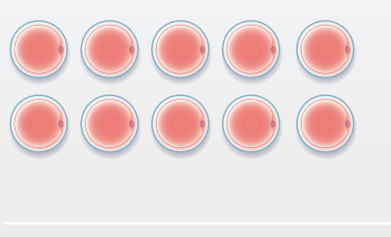

Seuls 6 ovules seront fécondés. Tous seront développés.

Durant 5 jours, les ovules fécondés se développeront sans intervention extérieure. 4 des 6 ovules arrêteront alors de se développer, comme c'est le cas naturellement.
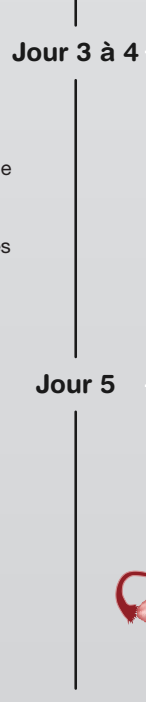

Le médecin implantera l'un des 2 embryons viables dans l'utérus; les autres seront congelés.
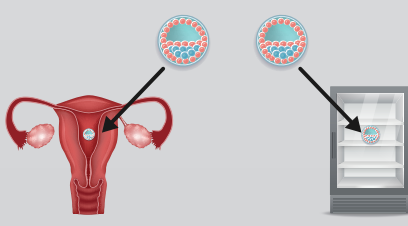

La nouvelle loi prévoit de diminuer massivement le nombre d'embryons congelés, réduisant ainsi de manière significative la durée du traitement.

* Toutes les données exprimées correspondent à des valeurs moyennes

Figure 1: La procréation médicalement assistée maintenant et avec la nouvelle loi (représentation mise à disposition des auteurs). et le jour 5. Comme ce processus abortif naturel se déroule longtemps avant le premier symptôme clinique habituel d'une grossesse, à savoir l'absence de menstruation, la femme n'a absolument pas conscience d'avoir été enceinte. C'est pourquoi ce processus est appelé avortement subclinique ou préclinique. Plus le temps d'observation de ce processus de développement naturel in vitro sera long, plus le nombre d'embryons imprégnés sera important et plus la chance d'obtenir un embryon viable susceptible d'induire une grossesse sera élevée. Cependant, il est aussi essentiel que ce processus d'observation ne devienne pas un processus de sélection active de l'embryologiste et du médecin traitant, mais qu'il reste un processus naturel du développement embryonnaire précoce. En comparaison à la législation actuelle, la révision de la LPMA permettra de réduire de manière substantielle le nombre de transferts d'embryon nécessaires au démarrage d'une grossesse. $\mathrm{La}$ durée de traitement et les coûts, qu'aucune assurance ne couvre et qui sont donc toujours à la charge du couple, seront ainsi considérablement réduits.

Afin de ne pas avoir à détruire les éventuels embryons viables restants, la cryoconservation d'embryons qui est une méthode pratiquée de façon routinière dans d'autres pays européens depuis de nombreuses années - serait désormais autorisée en Suisse (art. 17, al. 3 LPMA). Grâce à cela, il sera possible d'éviter à la patiente de nouveaux traitements de stimulation hormonale et prélèvements d'ovules lourds et onéreux. Comme une plus longue période d'observation entraîne une diminution massive des embryons surnuméraires, le nombre d'ovules développés qui sont stockés chaque année passerait de près de 16000 par an actuellement à moins de 8000 sous le régime de la LPMA révisée. C'est une baisse considérable et une conséquence bénéfique de la nouvelle législation sur le plan économique et sociétal.

\section{Réduire le nombre des grossesses multiples à risque}

Comme mentionné plus haut, une grande partie des embryons non viables dégénère naturellement entre le jour 1 et le jour 5 . Il sera donc beaucoup plus facile de déterminer au jour 5 l'embryon qui a le meilleur potentiel de développement sur les 12 embryons maximaux autorisés sur la base de critères morphologiques clairement définis, au lieu de devoir choisir au jour 1 à partir d'indications imprécises entre seulement 3 embryons imprégnés. Cette optimisation permet d'implanter un seul embryon vivant et en bon état au jour 5 au lieu de 2 à 3 embryons au jour 2 ou 3, ce qui implique, à chance égale de grossesse, un risque de grossesse multiple net- 
tement moins élevé. Cette technique appelée «transfert électif d'un seul embryon (eSET)» est utilisée avec succès en Suède, en Finlande et en Belgique depuis de nombreuses années [6]. Le transfert d'un seul embryon au jour 5 réduit considérablement les risques de grossesses multiples lors de la procréation médicalement assistée, sans pour autant affecter les chances de grossesse. Rappelons qu'une grossesse multiple représente un risque pour la mère et l'enfant, notamment de prématurité, et par conséquent un risque de décès et d'handicap pour l'enfant.

Tableau 1: Législation en vigueur dans I'Union européenne et en Suisse, état 2016; adaptée et actualisée selon [2].

\begin{tabular}{|c|c|c|c|c|}
\hline Méthode & SET & $\begin{array}{l}\text { Cryoconservation } \\
\text { des embryons }\end{array}$ & PGD & PGS \\
\hline Belgique & OUI & OUI & OUI & OUI \\
\hline Bulgarie & OUI & OUI & OUI & OUI \\
\hline Danemark & OUI & OUI & OUI & OUI \\
\hline Allemagne & En suspens & En suspens & OUI & En suspens \\
\hline Estonie & OUI & OUI & OUI & OUI \\
\hline Finlande & OUI & OUI & OUI & OUI \\
\hline France & OUI & OUI & OUI & NON \\
\hline Grèce & OUI & OUI & OUI & OUI \\
\hline Grande-Bretagne & OUI & OUI & OUI & OUI \\
\hline Irlande & OUI & OUI & OUI & OUI \\
\hline Italie & OUI & OUI & OUI & OUI \\
\hline Croatie & OUI & OUI & OUI & OUI \\
\hline Lettonie & OUI & OUI & OUI & OUI \\
\hline Lituanie & OUI & OUI & NON & NON \\
\hline Luxembourg & OUI & OUI & OUI & OUI \\
\hline Malte & OUI & OUI & OUI & OUI \\
\hline Pays-Bas & OUI & OUI & OUI & OUI \\
\hline Autriche & OUI & OUI & OUI & OUI \\
\hline Pologne & OUI & OUI & OUI & OUI \\
\hline Portugal & OUI & OUI & OUI & OUI \\
\hline Roumanie & OUI & OUI & OUI & OUI \\
\hline Suède & OUI & OUI & OUI & NON \\
\hline Slovaquie & OUI & OUI & OUI & OUI \\
\hline Slovénie & OUI & OUI & OUI & NON \\
\hline Espagne & OUI & OUI & OUI & OUI \\
\hline Tchéquie & OUI & OUI & OUI & OUI \\
\hline Hongrie & OUI & OUI & OUI & OUI \\
\hline Chypre & OUI & OUI & OUI & OUI \\
\hline Suisse & NON & NON & NON & NON \\
\hline
\end{tabular}

OUI: autorisé

NON: interdit

SET: Single Embryo Transfer

PGD: Preimplantation Genetic Diagnosis

PGS: Preimplantation Genetic Screening

\section{Eviter des interruptions de grossesse inutiles}

Le diagnostic préimplantatoire (DPI) est la méthode la plus précoce du diagnostic prénatal (DPN) invasif, un diagnostic généralisé et accepté depuis longtemps déjà par une grande partie de la population en Suisse. Avec le DPI, le couple n'est pas confronté, dans le cas d'un résultat défavorable au jour 5 , à la décision d'une interruption de grossesse, contrairement à ce qui est le cas avec le DPN qui implique une grossesse intacte et qui, selon la méthode, ne peut être pratiqué qu'au terme de la $11^{\mathrm{e}}$ semaine de grossesse.

La modification de l'art. 5, al. 3 et de l'art. 17, al. 1 et 3 de la LPMA ouvrira en Suisse la voie à un DPI judicieux tel qu'il est déjà pratiqué dans toute l'Europe, et depuis des décennies dans certains pays, à l'exception de la Lituanie et du Vatican. Cette vaste et longue expérience des pays européens environnants, qui a eu lieu sans aucun abus ni pression sur les couples ou les personnes handicapées, montre que le DPI peut être introduit en Suisse en toute confiance.

Contrairement au DPN, le DPI classe les différentes indications en deux groupes. Le premier comprend l'examen génétique d'un embryon afin de dépister des maladies héréditaires. Un DPI avec cette indication sera classé dans la terminologie scientifique PGD (Preimplantation Genetic Diagnosis). Le second groupe comprend l'examen génétique d'un embryon pour détecter des anomalies chromosomiques intervenues le plus souvent dans les ovules vieillissants et qui ont été transmises à l'embryon. Cet examen s'appelle Preimplantation Genetic Screening (PGS) et il est analogue au dépistage de la trisomie lors du DPN. Ainsi, toutes les analyses génétiques plus poussées, ainsi que les interventions sur le génome, demeurent absolument interdites par la nouvelle législation (art. 35 LPMA). C'est pourquoi il est non seulement trompeur, mais aussi complètement faux de laisser entendre que les PGS autorisés par la révision de la LPMA permettraient de créer des bébés «sur mesure». Le PGS est comparable aux milliers de dépistages de la trisomie effectués chaque année en Suisse dans le cadre d'un DPN, à la seule différence qu'il est avancé de la $11^{\mathrm{e}}$ semaine au jour 5 de grossesse.

A l'instar d'un DPN, un PGS n'est pas un examen de routine pratiqué sans la volonté expresse du couple. D'une part, ce dernier est informé du déroulement, des chances et des risques liés à son souhait et, d'autre part, il doit donner son consentement écrit à la mise en œuvre d'un PGS supplémentaire dans le cadre du traitement. C'est également le couple, et non son médecin ou un embryologiste, qui décide du destin des embryons examinés. Outre le fait que de nombreux couples qui sou- 
haitent concrétiser leur désir d'enfant n'opteront pas pour le PGS pour des raisons financières, car le DPI n'est pris en charge par aucune assurance en Suisse, seule une partie infime des personnes traitées, notamment pour des raisons médicales et méthodiques, sera concernée par ce type d'examen. En effet, le PGS s'adresse principalement aux femmes de plus de 37 ans, qui sont relativement peu nombreuses à suivre ce traitement et chez qui plus de 10 ovules ont pu être prélevés. Si l'on considère les limites financières et techniques, le nombre d'examens PGS effectués chaque année en Suisse s'élèvera au mieux à plusieurs centaines et non à minimum 6000 comme on le prétend fréquemment.

Il serait particulièrement choquant d'interdire un PGS aux couples porteurs d'une maladie héréditaire grave ayant décidé de ne pas transmettre leur maladie à leurs enfants grâce au PGD. Dans un tel cas, un PGD extrêmement coûteux permettrait d'exclure la maladie, par ex. une ostéogenèse imparfaite, mais un dépistage supplémentaire de la trisomie sur l'embryon serait en revanche interdit. Si le transfert d'un embryon non affecté par l'ostéogenèse imparfaite aboutit à une grossesse, le dépistage de la trisomie souhaité par les parents ne pourrait être effectué que dans la $11^{\mathrm{e}}$ semaine. Il serait alors probable qu'ils optent pour une interruption de grossesse. Sur le plan éthique, une telle réglementation serait inacceptable car le généticien réalisant le PGD aurait eu connaissance de l'anomalie chromosomique avant le transfert de l'embryon et donc avant la grossesse, mais n'aurait pas pu en informer ni le couple ni son médecin en raison des interdictions légales.

\section{C'est pourquoi nous devons voter encore une fois OUI le 5 juin 2016}

La SSGO gynécologie suisse s'engage en faveur d'une révision équilibrée et nuancée de la Loi fédérale sur la procréation médicalement assistée pour que les couples qui ont des difficultés à concrétiser leur désir d'enfant puissent trouver en Suisse le même niveau de traitement que dans le reste de l'Europe. Cette modification permettra notamment (a) d'optimiser la prise en charge de milliers de couples désirant un enfant, (b) de réduire fortement le nombre des grossesses multiples à risque après une procréation médicalement assistée et (c) d'éviter des interruptions de grossesse grâce à l'introduction du DPI qui est la méthode de diagnostic prénatal la plus précoce et la moins invasive.

\section{Références}

1 Assemblée fédérale de la Confédération helvétique. Loi fédérale sur la procréation médicalement assistée (LPMA) Confédération helvétique 1998/2006; RS 810.11:1-14.

2 Comparative Analysis of Medically Assisted Reproduction in the EU. MAR Study. ESHRE 2009;1-157.

3 Assemblée fédérale de la Confédération helvétique. Message concernant la modification de l'article constitutionnel relatif à la procréation médicalement assistée et au génie génétique dans le domaine humain. Confédération helvétique 2014;9675-6.

4 Assemblée fédérale de la Confédération helvétique. Loi fédérale sur la procréation médicalement assistée (LPMA). Confédération helvétique 2014;1-9.

5 Frommel M, Thaler Ch. 10 Jahre «deutscher Mittelweg». Frauenarzt 2015;56:14-6.

6 Källen B, Finnström O, Lindam A, Nilsson E, Nygren KG, Otterblad Olavsson P. Trends in delivery and neonatal outcome after in vitro fertilization in Sweden : data for 25 years. Hum Reprod 2010;25:1026-34. 